\title{
CLASSIFICAÇÃO DE CRISES EPILÉPTICAS DE CRIANÇAS COM BASE NA DESCRIÇÃO CLIINICA DOS PAIS OU RESPONSÁVEIS
}

\author{
Angélica Amorim Amato', Erik Choult' , Maria Claúdia Sampaio', \\ Carlos Nogueira Aucélio ${ }^{2}$, Áurea Nogueira de Melo ${ }^{3}$
}

\begin{abstract}
RESUMO - O objetivo deste estudo foi determinar com que frequência a descrição das manifestações epilépticas observadas pelos pais ou responsáveis por crianças com epilepsia permitem a classificação clínica das crises epilépticas(CE). Foram selecionadas 112 crianças com CE recorrentes e espontâneas. 0 estudo foi dividido em duas partes. Na Parte I avaliamos a idade na primeira crise, modo de início, semiologia pré-ictal, ictal e pós-ictal. Na Parte II a classificação das CE: a) segundo padrão motor convulsivo, em convulsivas / não convulsivas; e b) segundo o esquema da Liga Internacional Contra Epilepsia ( ILAE, 1981). Os resultados significantes foram a descrição de auras epilépticas em $42,9 \%$, sinais localizatórios em $36,6 \%$ e a descrição semiológica motora em $95,5 \%$, neurovegetativa em $56,3 \%$, psíquica em $32,1 \%$ e sensitivo-sensorial em $4,5 \%$. Foram classificadas pela semiologia motora as CE convulsiva em $83 \%$ e não convulsiva em $17 \%$. No esquema da ILAE obteve-se na amostra geral $12,5 \%$ de CE não classificadas, pela dicotomia foram classificadas as CE parciais em $59,9 \%$ e as generalizadas em $27,2 \%$. Um alto índice de aproveitamento das informações foi obtido com $87,5 \%$ de CE classificadas.Este resultado nos permite concluir ser possível classificar clinicamente e com segurança as crises epilépticas com base na descrição dos pais e ou responsáveis, utilizando-se protocolo padronizado.
\end{abstract}

PALAVRAS-CHAVE: epilepsia, crises epilépticas na criança, classificação, informações dos pais, sintomatologia.

\section{Classification of seizures in childhood obtained from seizures symptomatology descriptions of parents and guardians}

ABSTRACT - The aim of this study was to determine the frequency of seizures descriptions from parents and guardians of epileptic children which are useful for clinical classification of epileptic seizures. The data were obtained from 112 children using recurrent and spontaneous epileptic seizures as the selection criteria. The study was realized in two parts. In the part I the following aspects were studied: age of onset and the preictal, ictal and postictal symptoms. In the part II the specific kinds of seizures were classified by motor semiology, as convulsive and nonconvulsive, and by using the International League Against Epilepsy (ILAE,1981) scheme. The results showed that $42.9 \%$ patients present epileptic auras and $36.6 \%$ present lateralizing signs. Concerning the semiologic events were motor in $95.5 \%$, neurovegetative in $56.3 \%$, psychic in $32.1 \%$, and neurosensorials in $4.5 \%$. Finally, the ILAE schema classified the seizures as partial in $59.9 \%$, as generalized in $27.2 \%$, and as not classified in $12.5 \%$. All findings demonstrate a good level of clinical semiology information from parents and guardians that allow us to classify $87.5 \%$ of the seizures, and identify auras and lateralized signs. Theses results permit us to conclud that if a standard protocol is used, the descriptions from parents and guardian of epileptic children appears to be very reliable to clinical classification of epileptic seizure.

KEY WORDS: epilepsy, childhood epileptic seizure, classification, parents clinical discriptions, symptomatology.

Apesar de todo avanço tecnológico, principalmente dos video-EEG sincronizados e dos exames de neuroimagem, na investigação da epilepsia (EP) ${ }^{1-3}$ o seu diagnóstico em princípio continua a ser eminentemente clínico e dependente das informações na maior parte daqueles que assistem as crises epilépticas (CE). Deste ponto de vista, três partes são determinantes: 1) definir se o paciente tem EP, 2) classificar o tipo de CE e 3) definir a etiologia de base.

A classificação da CE é fundamental não somente para confirmar o diagnóstico de EP mas também como guia importante para escolha da droga inicial

\footnotetext{
Faculdade de Ciências da Saúde(FCS) / Hospital Universitário(HUB) da Universidade de Brasília (UnB), Brasilia DF, Brasil: ${ }^{1}$ Aluno da Graduação do Curso Médico da FCS; ${ }^{2}$ Mestre Professor Adjunto de Neurologia Infantil, Departamento de Pediatria da FCS / HUB-UnB e Centro de Ciências da Saúde(CCS) da Universidade Federal do Rio Grande do Norte (UFRN) Natal RN, Brasil; ${ }^{3}$ Doutora Professora Adjunta de Neurologia Infantil, Departamento de Pediatria e Medicina Clínica do CCS - UFRN.
}

Recebido 23 Julho 2002, recebido na forma final 27 Novembro 2002. Aceito 9 Dezembro 2002.

Dra. Áurea Nogueira de Melo - Rua Paulo Lyra 2183/1301 - 59064-550 Natal RN - Brasil.E-mail:aurea@ccs.ufrn.br 
e para a formulação do prognóstico ${ }^{4}$. Raramente os neurologistas têm a oportunidade de assistir a uma $\mathrm{CE}$, e com isso sua caracterização depende das informações do paciente quando possível ou, na maioria das vezes, de outrem ${ }^{5}$. Nas crianças com EP as CE são descritas pelos pais ou responsáveis $(\mathrm{P} / \mathrm{R})$ e as observações relatadas poderão ou não contribuir para a classificação correta das manifestações epilépticas ${ }^{6-9}$.

O presente estudo tem como objetivo avaliar o perfil e determinar a frequência das informações dos $P / R$ de crianças com EP que permitem a classificação clínica das $\mathrm{CE}$, obtida de um protocolo padronizado.

\section{MÉTODO}

Foram selecionadas 112 crianças com o diagnóstico de EP do Ambulatório de Neuroepilepsia da Criança do Hospital Universitário de Brasília, de 1998 a 2000, na faixa etária de 6 meses a 14 anos; 62 crianças eram do genero masculino e 50 do feminino. Os critérios de seleção foram: 1) ter diagnóstico de EP (mais de duas CE recorrentes e espontâneas ${ }^{10}$ ); 2 ) mais de três atendimentos ambulatoriais por ano; 3 ) informações completas sobres as $C E$ obtidas dos $\mathrm{P} / \mathrm{R}$ a partir de um protocolo padronizado. Os protocolos foram preenchidos durante o atendimento ambulatorial por dois neurologistas infantis. $O$ estudo das características semiológicas das CE obedeceu rigorosamente à sequência dos eventos pré-ictal, ictal e pós-ictal. Foram consideradas auras epilépticas quando o $P / R$ respondeu prontamente e com segurança que sabia quando a criança iria apresentar a $\mathrm{CE}$, porque a criança relatava o que estava sentindo ou pela observação de alterações precedendo as CE. Foram considerados sinais localizatórios os eventos relatados espontaneamente pelos $\mathrm{P} / \mathrm{R}$ e observados durante as $\mathrm{CE}$ como: versão cefálica e ocular, desvio da boca, postura distônica, automatismos unilaterais e movimentos tônicos ou clônicos lateralizados.

$A$ avaliação do perfil das informações dos $P / R$ foram divididas em duas partes. Na parte I as seguintes variáveis foram estudadas: idade na primeira $\mathrm{CE}$, modo de início, semiologia pré-ictal, ictal e pós-ictal. Na parte II foi avaliada a classificação das CE dividindo-as em duas categorias. Na categoria I, as CE foram classificadas tendo como base a semiologia motora convulsiva: $\mathrm{CE}$ convulsivas versus CE não convulsivas. Na categoria II, as CE foram classificadas com base no esquema de classificação internacional da International League Against Epilepsy (ILAE, $1981^{11}$ ) em três etapas: Etapa I - segundo a dicotomia parcial versus generalizada; Etapa II- subclassificação das CE em parciais (II-A) e generalizadas (II-B); e Etapa III - caracterização semiológica das crises parciais simples (III-A) e complexas (III-B). Utilizamos a estatística descritiva para análise dos dados, quantificando as informações obtidas dos $\mathrm{P} / \mathrm{R}$ assim como a classificação clínica das $\mathrm{CE}$, com o objetivo de determinarmos as frequências relativas em valores absolutos e percentuais.

\section{RESULTADOS}

As informações dos P/R nos casos estudados foram obtidas das mães em $66 \%$, dos pais em $4,5 \%$, de ambos em $3,6 \%$ e de outros em $5,4 \%$. A idade na primeira CE foi de $6 \mathrm{~m}$ a 14 anos, com o pico na faixa etária de 6 meses a 3 anos. $O$ modo de início das CE foi súbito em $66 \%$, lento em $6,3 \%$ e não registrado em $27,7 \%$.

Na quantificação da semiologia das CE na fase pré-ictal os $\mathrm{P} / \mathrm{R}$ descreveram auras epilépticas em 47 $(42 \%)$ casos e em 65 (56,2\%) elas não foram observadas. A faixa etária das crianças com auras foi dos 2 anos e 8 meses a 14 anos de idade. Em treze casos foi descrito um único tipo de aura epiléptica e em 35 casos mais de um tipo. A cefaléia foi a manifestação mais descrita como aura. Com base nas descrições dos $\mathrm{P} / \mathrm{R}$ os principais tipos de auras classificadas foram: comportamental em 26 casos, neurovegetativa em 25 casos e sensorial em 18 casos (Tabela 1).

$\mathrm{Na}$ fase ictal os $\mathrm{P} / \mathrm{R}$ descreveram sinais lateralizados em 41 (36,6\%) pacientes e em 71 (63,3\%) eles não foram observados. Em 24 casos os sinais eram isolados e em 17 os sinais eram associados. 0 sinal mais observado foi o desvio-oculocefálico em 16 $(39,9 \%)$ pacientes (Tabela 2) .Os principais eventos semiológicos observados nesta fase foram: motores em 100 (95,5\%), neurovegetativos em 63 (56,3\%), psíquicos em $36(32,1 \%)$ e sensitivo-sensoriais em 51 (4,5\%). Quando separamos a semiologia ictal em convulsiva versus não convulsiva, os fenômenos convulsivos foram descritos em 93 (83\%) pacientes e não convulsivos em 19 (17\%).

$\mathrm{Na}$ fase pós-ictal os $\mathrm{P} / \mathrm{R}$ descreveram eventos que sucediam as CE em 47 (42\%) pacientes e em 65 (58\%) casos eles estavam ausentes. Em ordem decrescente os principais eventos observados foram: sonolência $(100 \%)$, cefaléia $(38,2 \%)$, liberação esfincteriana (32\%), choro $(19,1 \%)$, confusão mental $(19,1 \%)$, vômitos $(17 \%)$, sono $(14,8 \%)$, irritabilidade $(6,4 \%)$, anorexia $(6,4 \%)$, sialorréia $(6,4 \%)$ e outros $(2,1 \%)$.

Na parte II as informações dos P/R na categoria I foi possível classificar $100 \%$ das CE: convulsivas em $83 \%$ e não convulsivas em $17 \%$. Nas não convulsivas em 7 casos a descrição semiológica eram típicas de CE hipomotoras. Na categoria II com base na classificação da ILAE, na etapa I foi possível classificar: CE parciais em 67 (58,8\%) casos, CE generalizadas em 31 (7,7\%) casos, e não classificadas em 14 (12,5\%). $\mathrm{Na}$ etapa II a sub-classificação das CE parciais (II-A), classificamos 57 (85,6\%) casos e em 10 casos (14,9\%) elas não foram classificadas. Nas generalizadas (IIB) todos os casos foram classificados. Na etapa III todos os tipos de CE parciais simples e complexas 
Tabela 1. Principais tipos de auras epilépticas observadas pelos pais ou responsáveis por 42 pacientes, atendidos no Ambulatório de Neuroepilepsia do Hospital Universitário de Brasília.

Tipo de Aura Número Freqüência

(\%)

\begin{tabular}{|c|c|c|}
\hline \multicolumn{3}{|l|}{ Neurovegetativa (25) } \\
\hline Palidez & 7 & 6,3 \\
\hline Sudorese & 7 & 6,3 \\
\hline Cianose & 3 & 2,7 \\
\hline Taquicardia & 3 & 2,7 \\
\hline Piloereção & 3 & 2,7 \\
\hline Rubor Facial & 1 & 0,9 \\
\hline Apnéia & 1 & 0,9 \\
\hline \multicolumn{3}{|l|}{ Comportamental (23) } \\
\hline Choro & 6 & 5,3 \\
\hline Irritabilidade & 5 & 4,4 \\
\hline Gemidos & 3 & 2,7 \\
\hline Agitação psicomotora & 2 & 1,8 \\
\hline Alteração do humor & 2 & 1,8 \\
\hline Desatenção & 1 & 0,9 \\
\hline Apatia & 1 & 0,9 \\
\hline Gritos & 1 & 0,9 \\
\hline \multicolumn{3}{|l|}{ Sensorial (18) } \\
\hline Cefaléia & 10 & 8,9 \\
\hline Parestesias & 2 & 1,8 \\
\hline Dor Abdominal & 2 & 1,8 \\
\hline Dor em Membros & 1 & 0,9 \\
\hline Disestesias & 1 & 0,9 \\
\hline Epigastralgia & 1 & 0,9 \\
\hline Sensação de "pernas pesadas" & 1 & 0,9 \\
\hline \multicolumn{3}{|l|}{ Gástrica (9) } \\
\hline Vômitos & 5 & 4,4 \\
\hline Naúseas & 4 & 3,6 \\
\hline \multicolumn{3}{|l|}{ Vertiginosa (5) } \\
\hline Tonturas & 5 & 4,4 \\
\hline \multicolumn{3}{|l|}{ Visual (4) } \\
\hline Escotomas & 2 & 1,8 \\
\hline Alterações visuais & 2 & 1,8 \\
\hline Amaurose & 1 & 0,9 \\
\hline Fotofobia & 1 & 0,9 \\
\hline \multicolumn{3}{|l|}{ Motora ocular (1) } \\
\hline Oculógiro & 1 & 0,9 \\
\hline
\end{tabular}

foram classificadas. Na Tabela 3 detalhamos os achados da categoria II. O perfil da classificação na amostra geral tendo como enfoque a dicotomia CE classificadas versus não classificadas, as informações $P / R$ permitiram classificar $87,5 \%$ dos pacientes selecionados (Tabela 4).
Tabela 2. Principais sinais localizatórios descritos pelos pais ou responsáveis por 41 crianças com epilepsia, atendidas no Ambulatório de Neuroepilepsia do Hospital Universitário de Brasília.

\begin{tabular}{lll}
\hline Sinais & Número & Percentagem \\
localizatórios & de casos & \\
\hline
\end{tabular}

\section{Isolados (24)}

Tônico focal

Desvio cefálico

19,5

Clônico focal

14,6

Desvio ocular

12,1

Desvio da comissura labial

7,3

4,8

Associados (17)

Desvio oculocefálico/

17,0

hipertonia focal

Desvio oculocefálico

Desvio oculocefálico/

3

comissura labial

Desvio cefálico / clônico facial

1

14,6

7,3

2,4

\section{DISCUSSÃO}

A avaliação dos resultados mostra alto índice de frequência de informações positivas de um protocolo padronizado para EP da criança obtidas dos P/ $\mathrm{R}$, permitindo com relativa segurança classificar clinicamente as CE. Este achado é semelhante aos descritos por Oostrom et al. ${ }^{12}$ quando relataram as percepções dos pais em relação a epilepsia de suas crianças. Ainda, dois achados importantes sustentam esta afirmação: a descrição de auras epilépticas e a identificação de sinais localizatórios pelos P/R. Este alto índice de informações positivas, que permitiu a classificação clínica das $\mathrm{CE}$, também é explicado: pelo fato do estudo ter sido realizado utilizando-se um protocolo padrão próprio para EP; e pela entrevista pessoal dos investigadores neurologistas infantis, evitando a falta de dados sobre as CE. Isso pode dificultar a classificação, como relatado por Goodridge e Shorvon, $1983^{13}$. Na literatura, Picot et al. ${ }^{14}$, em estudo de pacientes adultos com epilepsia, relatam a validade da entrevista semi-estruturada para a classificação de CE.

A maioria nas informações foi obtida das mães em função da estrutura familiar em nosso país, que reflete a alta responsabilidade que elas têm pela saúde de suas crianças.

A idade na primeira CE só não foi determinada em $2 \%$ dos pacientes, face à falta de segurança dos $\mathrm{P} / \mathrm{R}$ em afirmar categoricamente $\mathrm{a}$ idade precisa.

Na avaliação da fase pré-ictal, a observação mais surpreendente foi a descrição de auras epilépticas. 
Tabela 3. Classificação das crises epilépticas de 112 crianças com epilepsia a partir das informações dos pais ou responsáveis, segundo a ILAE, atendidas no Ambulatório de Neuroepilepsia do Hospital Universitário de Brasília.

\begin{tabular}{|c|c|}
\hline Etapas & Número (\%) \\
\hline \multicolumn{2}{|l|}{ Etapa I } \\
\hline Parcial & $67(59,8)$ \\
\hline Generalizada & $31(27,7)$ \\
\hline Não classificadas & $14(12,5)$ \\
\hline \multicolumn{2}{|l|}{ Etapas II - A (caracterização das crises parciais) } \\
\hline Parcial simples & $11(16,4)$ \\
\hline Parcial complexa & $7(10,4)$ \\
\hline $\begin{array}{l}\text { Parcial simples evoluindo para } \\
\text { parcial complexa }\end{array}$ & $1(1,5)$ \\
\hline $\begin{array}{l}\text { Parcial simples com generalização } \\
\text { secundária }\end{array}$ & $30(44,7)$ \\
\hline $\begin{array}{l}\text { Parcial complexa com generalização } \\
\text { secundária }\end{array}$ & $5(7,4)$ \\
\hline $\begin{array}{l}\text { Parcial simples } \rightarrow \text { complexa } \rightarrow \text { generalização } \\
\text { secundária }\end{array}$ & $3(5,9)$ \\
\hline Não classificadas & $10(14,4)$ \\
\hline \multicolumn{2}{|l|}{ Etapa II - B (caracterização das crises generalizadas) } \\
\hline Tônico-clônicas & $16(51,6)$ \\
\hline Clônicas & $4(12,9)$ \\
\hline Tônicas & $3(9,7)$ \\
\hline Ausências & $3(9,7)$ \\
\hline Espasmos Infantis & $3(9,7)$ \\
\hline Mioclônica-astática & $2(6,4)$ \\
\hline \multicolumn{2}{|l|}{ Etapa III - A (semiologia das crises parciais simples) } \\
\hline Motoras & $29(67,4)$ \\
\hline Neurovegetativas & $26(63,4)$ \\
\hline Sensitivas & $11(26,8)$ \\
\hline Psiquicas & $10(24,4)$ \\
\hline Sensoriais & $7(17,1)$ \\
\hline Hipomotoras & $7(17,1)$ \\
\hline \multicolumn{2}{|c|}{ Etapa III - B (semiologia das crises parciais complexas) } \\
\hline $\begin{array}{l}\text { Com alterações da consciência } \\
\text { desde o início }\end{array}$ & $12(75,5)$ \\
\hline $\begin{array}{l}\text { 1) Apenas com alterações } \\
\text { da consciência }\end{array}$ & $3(18,7)$ \\
\hline 2) Com automatismos & $9(56,3)$ \\
\hline $\begin{array}{l}\text { Início parcial simples } \rightarrow \text { alteração } \\
\text { da consciência }\end{array}$ & $4(25,0)$ \\
\hline
\end{tabular}

ILAE, International League Against Epilepsy (Epilepsy 1981; 22: 498-501); $\rightarrow$ evoluindo para.

Identificar auras do ponto de vista clínico tem grande importância, pois permite separar as CE parciais secundariamente generalizadas das generalizadas primárias. Como relataram Acharya et al. ${ }^{15}$ e Henkel et al. ${ }^{16}$, identificar auras em crianças é uma importante informação para definir seu início localizado.
Tabela 4. Perfil final da classificação das Crises Epilépticas de 112 crianças com Epilepsia segundo as informações obtidas dos pais ou responsáveis atendidas no Ambulatório de Neuroepilepsia do Hospital Universitário de Brasília.

\begin{tabular}{lcc}
\hline Classificação & Classificadas & Não Classificadas \\
\hline Geral & $87,5 \%$ & $12,5 \%$ \\
Dicotomia & & \\
$\quad$ Parciais & $59,9 \%$ & $27,2 \%$ \\
Generalizadas & $100 \%$ & - \\
Semiologia parcial & & \\
Parcial simples & $100 \%$ & - \\
Parcial complexa & $100 \%$ & - \\
\hline
\end{tabular}

Estes achados são divergentes dos de Bodersteiner et al. ${ }^{17}$ que estudaram 1883 crianças avaliando a variabilidade da classificação de $C E$, segundo a ILAE; eles não relatam auras em seus resultados. Hamer et al. ${ }^{18}$ não encontraram descrição de auras em crianças abaixo de 3 anos de idade, estudando CE por video-EEG. A predominância da cefaléia como aura na criança é concordante com os achados descritos por Karaali-Savrun et al. ${ }^{19}$, quando descreveram a cefaléia peri-ictal em pacientes com epilepsia.

$\mathrm{Na}$ fase ictal foi possível identificar sinais localizatórios em $36,6 \%$, que ajudaram na classificação das CE parciais com generalização secundária. Os achados predominantes foram desvio óculo-cefálico e movimentos localizados tônicos ou clônicos de membros. Estes achados são totalmente condizentes com os de Chee et al. ${ }^{20}$, Willie et al. ${ }^{21}$ e Olbrich et al. ${ }^{22}$, quando estudaram sinais localizatórios nas CE utilizando o video-EEG. São concordantes ainda, com os de Maclachlan ${ }^{23}$, que descreveu sinais focais tipo clônicos tendo como base a avaliação por questionários. Embora Lüders et al. ${ }^{24,25}$ afirmem que definir a localização de CE pela sua semiologia focal é importante principalmente para pacientes candidatos a cirurgia, ressaltamos que também é fundamental para os casos não cirúrgicos, pois o prognóstico e a resposta terapêuti-

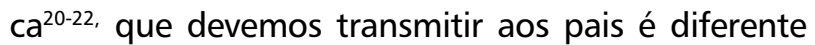
quando a EP é rolândica ${ }^{26}$, occipital ${ }^{27}$ ou frontal ${ }^{28}$.

Os fenômenos motores convulsivos foram os mais observados pelos $P / R$, e este achado é perfeitamente entendido pelo fato destes movimentos involuntários representarem eventos aberrantes e terríveis para leigos que os assistem, mesmo quando a CE é motora focal. Justifica-se ainda pela sua semiologia mais exuberante e assustadora, principalmente quando generalizada, o que permitiu ser mais observada e lembrada pelos P/R. Estes achados da categoria I 
são concordantes com os de Wulf5. Este autor avaliou a observação e a documentação de CE usando video-tape: as CE com excesso de atividade motora obtiveram alto escore de observação. Na classificação de CE não convulsivas, as descrições de sete casos nos permitiram classificá-las como CE hipomotoras. Estas CE hipomotoras foram bem detalhadas por Acharya et al. ${ }^{15}$ que, através de video-EEG, confirmaram a descrição relatada pela mãe. Este dado da literatura nos faz acreditar na validade das observações feitas pelos P/R em nosso estudo. Este tipo de $\mathrm{CE}$, embora seja reconhecidamente parcial, não foi catalogada na classificação da ILAE,1981'11. No recente esquema de classificação proposto pela ILAE$2001^{29}$, as CE hipomotoras foram incorporadas na classificação das CE focais. Chamamos a atenção que as $\mathrm{CE}$ hipomotoras descritas pelos $\mathrm{P} / \mathrm{R}$ seguramente podem ser confundidas com crises generalizadas primárias, secundariamente generalizadas ou serem mal interpretadas como estado pós-ictal.

Na etapa I, com base na dicotomia, as CE não foram classificadas em $12,5 \%$ dos casos. Este dado é comparável aos de Goodridge e Showon ${ }^{13}$, que relatam 7\% de CE não classificadas ao estudarem 122 crianças com CE. Esta frequência é bem menor quando comparada à de Bodensteiner et al. ${ }^{17}$ que, em sua amostra de 1883 pacientes, relatam que em $22 \%$ dos seus casos as CE não foram classificadas. Na etapa II, com base na subclassificação, não foi possível classificar $14,4 \%$ das CE parciais. Este índice indica limitação do uso da dicotomia parcial simples versus complexa com base no envolvimento ou não da consciência. Acharya et al. ${ }^{15}$, estudando CE parciais em crianças por video-EEG e com concomitante observação pessoal, relatam que em alguns casos não foi possível definir com segurança o envolvimento ou não da consciência, dificultando a sub-classificação das crises parciais. Nas CE generalizadas, todos os casos foram classificados. Estes achados são explicados pelo fato de que na sub-classificação dessas CE o aspecto semiológico é mais importante do que o envolvimento ou não da consciência.

Outro dado surpreendente foi a classificação clínica de todas as CE parciais com base nos eventos semiológicos descritos pelos P/R. Este resultado nos faz propor valorização da semiologia das CE para sua classificação, maior do que simplesmente sua relação com início da descarga epiléptica, se focal ou generalizada. Lüders et al. ${ }^{24,25}$ propuseram a valorização da descrição semiológica, argumentando que ela contém muitas informações importantes e que são negligenciadas a favor de prioridade maior do envolvimento ou não da consciência. Recentemente, Parra et al. ${ }^{30}$, comparando os dois sistemas de classificação das CE com base semiológica e com o da ILAE-1981 ${ }^{11}$, ressaltam a utilidade e precisão da abordagem semiológica para classificação das crises epilépticas.

Na epileptologia moderna da década dos anos 90, a classificação das CE tem como base as informações clínicas associadas aos achados EEG, associada por vezes ao uso de tecnologias sofisticadas de monitorização de EEG para sua melhor definição e classificação. Entretanto, os resultados deste estudo em que as CE foram classificadas clinicamente tendo como suporte as descrições das manifestações epilépticas observadas pelos $P / R$ nos permitem as seguintes conclusões: 1) é possível obter alto índice de aproveitamento das observações colhidas através de um protocolo padronizado para EP; 2 ) os $\mathrm{P} / \mathrm{R}$ prestam informações com detalhes permitindo a identificação de auras e sinais focais; 3 ) há limites na classificação da ILAE-1981 no tocante às auras epilépticas não tendo sido as crises hipomotoras incorporadas a esta classificação; 4) o uso de protocolo padronizado para atendimento ambulatorial de crianças com EP permite classificação clínica mais precisa do tipo de CE proporcionando, a priori, a oportunidade de uma indicação clínica adequada da primeira droga antiepiléptica a ser prescrita .

\section{REFERÊNCIAS}

1. Lagerlund TD, Cascino GD, Cicora KM, Sharbrough FG. Long-term electroencephalographic monitoring and management of seizures. Mayo Clin Proc 1996;71:1000-1006.

2. Engel J Jr. Classification of the International League Against Epilepsy: time for reappraisal. Epilepsia 1998;39:1014-1017.

3. Gaillard WD. Structural and functional imaging in children with partial epilepsy. Ment Retard Dev Disabil Res Rew 2000;6:220-226.

4. Thurston JH, Thurston DL, Hixon BB, Keller AJ. Prognosis in childhood epilepsy: additional follow-up of 148 children 15 to 23 years after withdrawal of anticonvulsivant therapy. N Eng J Med 1982;306: 83 1- 836.

5. Wulf JA. Evaluation of seizures observation and documentation. J Neurosci Nurs 2000;32:27-36.

6. Norzila MZ, Azizi BH, Motila R. Childhood epilepsy: what parents know, believe and do. Med J Malaysia 1997;52:60-63.

7. Brouwer OF. Epileptic seizure in children. Ned Tijdschr Geneeskd 1999; 143:190-193.

8. Bautista JF, Lüders HO. Semiological seizure classification: relevance to pediatric epilepsy. Epilepsy Disord 2000;2:65-72.

9. Ried S, Hilfiker P, Mothersill I W, Kramer G. From clinical observation to long-term monitoring: diagnostic developments in conservative epileptology. Epilepsia 2000;41(Suppl 3):S2-9.

10. Gastaut H. Dictionary of epilepsy. Part I. Definitions. Geneva: Word Health Organization, 1973.

11. Commission on Classification and Terminology of the International League Against Epilepsy: proposal for revised clinical and electroencephalographic classification of epileptic seizures. Epilepsy 1981;22:498-501.

12. Oostrom KJ, Schouten A, Kruitwagen CL, Peters AC, JennekensSchinkel A; The Dutch Study Group of Epilelsy in Childhood. Parents' perceptions of adversity introduced by upheaval and uncertainty at onset of childhood epilepsy. Epilepsy 2001;42:1452-1460.

13. Goodrige DMG, Shorvon SD. Epileptic seizures in a population of 6000. I. Demography, diagnosis and classification, and role of the hospital service. Br Med J 1983;287:641-644. 
14. Picot MC, Crespel A, Tricot M, et al. Validity of diagnosis using the French translation of the semi-structured interview for seizure classification. Epilepsia 1999; 40:1649-1656.

15. Acharya JN, Wyllie E, Lüders HO, Kotagal P, Lancman M, Coelho MS. Seizure symptomatology in infants with localization-related epilepsy. Epilepsy 1997;48:189-196.

16. Henkel A, Noachtar S, Pfander M, Lüders HO. The localizing value of the abdominal aura and its evolution: a study in focal epilepsies. Neurology 2002;58:271-276.

17. Bodensteiner JB, Brownsworth RD, Knapik JR, Kanter CM, Cowan LD, Leviton A . Interobserver variability in the ILAE classification of seizures in childhood. Epilepsy 1988;29:123 -128.

18. Hamer HM, Wyllie E, Lüders HO, Kotagal P, Acharya J. Symptomatology of epileptic seizures in the first three years. Epilepsia 1999;40:837-844.

19. Karaali-Savrun F, Goksan B, Yeni SN, Ertan S, Uzun N. Seizurerelated headache in patients with epilepsy. Seizure 2002;11:67-69.

20. Chee MWL, Kotagal P, Van Ness PC, Gragg L Murphy D, Lüder HO. Lateralizing signs in intractable partial epilepsy: blided multipleobserver analysis. Neurology 1993;43:2519-2525.

21. Wyllie E, Lüders HO, Morris HH, Lesser RP, Dinner DS. The lateralizing significance of versive head and eye movements during epileptic seizures. Neurology 1986;36:606-611.

22. Olbrich A, Urak L, Groppel G, et al. Semiology of temporal lobe epilepsy in children and adolescents value in lateralizing the seizure onset zone. Epilesy Res 2002;48:103-110.
23. Mclachlan RS. The significance of head and eye turning in seizures. Neurology 1987;37:1617-1919.

24. Lüders HO, Burgess R, Noachtar S. Expanding the International Classification of Seizures to provide localization information. Neurology 1993;43:1650-1655.

25. Lüders HO, Acharya J, Baumgartner C, et al. A new epileptic seizure classification based exclusively on ictal semiology. Acta Neurol Scand 1999;99:137-141

26. Loiseau P, Duché B, Cordova S, Dartigues JF, Cohadon S. Prognosis of benign childhood epilepsy with centro-temporal spikes: a followup study of 168 patients. Epilepsia 1988;29:229-235.

27. Panayiotopoulos CP. Benign childhood e epileptic syndromes with occipital spikes: new classification proposed by the International League Against Epilepsy. J Child Neurol 2000;41:1139-1152.

28. Jobst BC, Siegel AM, Thadani VM, Roberts DW, Rhodes HC. Intractabel seizures of frontal lobe origin: clinical characteristic, localizing signs, and results of surgery. Epilepsia 2000;41:1139-1152.

29. Engel J Jr. A proposed diagnostic scheme for people with epileptic seizures and with epilepsy: report of the ILAE Task Force on Classification and Terminology. Epilepsia 2001;42:796-803.

30. Parra J, Augustijn PB, Geerts Y, van Emde Boas W. Classification of epileptic seizures: a comparation of two systems. Epilepsia 2001;42: 476-482. 